\title{
Functionals on Banach Algebras with Scattered Spectra
}

\author{
by \\ H. S. MUSTAFAYEV \\ Presented by Andrzej HULANICKI
}

Summary. Let $A$ be a complex, commutative Banach algebra and let $M_{A}$ be the structure space of $A$. Assume that there exists a continuous homomorphism $h: L^{1}(G) \rightarrow A$ with dense range, where $L^{1}(G)$ is a group algebra of the locally compact abelian group $G$. The main results of this note can be summarized as follows:

(a) If every weakly almost periodic functional on $A$ with compact spectra is almost periodic, then the space $M_{A}$ is scattered (i.e., $M_{A}$ has no nonempty perfect subset).

(b) Weakly almost periodic functionals on $A$ with compact scattered spectra are almost periodic.

(c) If $M_{A}$ is scattered, then the algebra $A$ is Arens regular if and only if $A^{*}=\overline{\operatorname{span}} M_{A}$.

1. Introduction. Throughout the paper $A$ will denote a complex, commutative Banach algebra. We shall denote by $M_{A}$ the structure space of $A$. As is well known, $M_{A}$ is a locally compact, Hausdorff space and the Gelfand transform $\Gamma: a \mapsto \widehat{a}$ identifies $A$ with a subalgebra of $C_{0}\left(M_{A}\right)$, the Banach algebra of all complex-valued continuous functions on $M_{A}$ which vanish at infinity. For $\varphi \in A^{*}$ and $a \in A$, the functional $\varphi \cdot a$ on $A$ is defined by $\langle\varphi \cdot a, b\rangle=\langle\varphi, a b\rangle, b \in A$. This operation turns $A^{*}$ into a Banach $A$-module. Let $O_{*}(\varphi)$ denote the weak*-closure of the set $\{\varphi \cdot a: a \in A\}$. Recall that the $w^{*}$-spectrum of a functional $\varphi \in A^{*}$, written $\sigma_{*}(\varphi)$, is defined by $O_{*}(\varphi) \cap M_{A}$. We can readily see that $\sigma_{*}(\varphi)=\operatorname{hull}\left(I_{\varphi}\right)$, where $I_{\varphi}=\{a \in A: \varphi \cdot a=0\}$ is a closed ideal in $A$.

Let $G$ be a locally compact abelian group and $L^{1}(G)$ be the group algebra of $G$. The well known Loomis theorem [9] states that if the $w^{*}$-spectrum of $\varphi \in L^{\infty}(G)$ is compact and scattered, then $\varphi$ is an almost periodic function, namely $\varphi \in \overline{\operatorname{span}} \sigma_{*}(\varphi)$. Recall that a closed subset $S$ of a topological

2000 Mathematics Subject Classification: 43A20, 43A60, 46J99.

Key words and phrases: Banach algebra, group algebra, (weakly) almost periodic functional, scattered set. 
Hausdorff space is said to be scattered if $S$ does not contain a nonempty perfect subset.

For $1<p<\infty$, let $A_{p}(G)$ denote the space of functions on $G$ which can be represented as

$$
f=\sum_{n=1}^{\infty} u_{n} * v_{n}^{\vee},
$$

where the $u_{n}$ 's are in $L^{p}(G)$, the $v_{n}$ 's are in $L^{q}(G)(1 / p+1 / q=1), v_{n}^{\vee}(g)=$ $v_{n}(-g)$, and

$$
\sum_{n=1}^{\infty}\left\|u_{n}\right\|_{p}\left\|v_{n}\right\|_{q}<\infty .
$$

The norm of $f$ is the infimum of the above sums over all such representations of $f$. The space $A_{p}(G)$ is a commutative Banach algebra [4], often called the Herz algebra. We recall that $L^{1}(\widehat{G})$ is isometrically isomorphic to $A_{2}(G)$ via the Fourier transform $F$. Here $\widehat{G}$ is the dual group of $G$. A generalization of the Loomis theorem to Herz algebras has been proved by Lust-Piquard in $[10]$.

As functions which are continuous on $G$ with compact support are dense in $L^{p}(G), A_{2}(G)$ is dense in $A_{p}(G)$. It follows that $F: L^{1}(\widehat{G}) \rightarrow A_{p}(G)$ is a continuous homomorphism with dense range. This suggests the question: Assume that there exists a continuous homomorphism $h: L^{1}(G) \rightarrow A$ with dense range. Is there a generalization of the Loomis theorem to the algebra $A$ ? In this paper we give a partial answer to this question.

We first note that the class of Banach algebras $A$ satisfying the above conditions is fairly large. In general, these algebras arise in the following way: Let $g \mapsto T_{g}$ be a bounded continuous representation of $G$ on a Banach space $X$. For $f \in L^{1}(G)$, define $T_{f}=\int_{G} f(g) T_{g} d g$. We see that $T_{f}$ is a bounded linear operator on $X$. Let $L_{T}(G)$ denote the closure of $\left\{T_{f}: f \in L^{1}(G)\right\}$ with respect to the operator-norm topology. Then the algebras $L_{T}(G)$ satisfy the conditions imposed on $A$.

If there exists a continuous homomorphism $h: L^{1}(G) \rightarrow A$ with dense range, the spectrum of $h$, written $\operatorname{sp}(h)$, is defined as the hull of the ideal $\operatorname{ker}(h)$. The standard technique of Banach algebras shows that $h^{*}$ homeomorphically identifieds $M_{A}$ with $\operatorname{sp}(h)$. Moreover, the Gelfand transform of $h(f)$ is just $\widehat{f}(\chi)(\chi \in \operatorname{sp}(h))$, where $\widehat{f}$ is the Fourier transform of $f \in L^{1}(G)$. If $\varphi \in A^{*}$, for notational simplicity we will write $h^{*} \varphi$ for $\varphi^{\vee}$. We shall also need the following notations: $X$ is a Banach space, $X^{*}$ is its dual, $X^{* *}$ is its second dual, and $X_{1}$ is the closed unit ball in $X$. We shall regard $X$ as naturally embedded into $X^{* *}$. For $\varphi \in X^{*}$ and $x \in X$, by $\langle\varphi, x\rangle$, and also by $\varphi(x)$, we denote the natural duality between $X^{*}$ and $X$. We will denote by $w$ and $w^{*}$ the weak topology in $X$ and the weak ${ }^{*}$ topology in $X^{*}$, respectively. By $\bar{E}^{w}$ and $\bar{E}$ we will denote the weak closure and the norm 
closure, respectively, of a set $E \subset X . \bar{E}^{*}$ will denote the weak ${ }^{*}$ closure of a set $E \subset X^{*}$.

2. Preliminaries. Let $A$ be a complex, commutative Banach algebra. The functional $\varphi \in A^{*}$ is said to be (weakly) almost periodic on $A$ if the set $\left\{\varphi \cdot a: a \in A_{1}\right\}$ is relatively (weakly) compact. This is equivalent to saying that the linear operator $T_{\varphi}: A \rightarrow A^{*}$ defined by $T_{\varphi}(a)=\varphi \cdot a$ is (weakly) compact. For example, if $A=L^{1}(G)$ then this reduces to the classical notion of (weak) almost periodicity for $\varphi \in L^{\infty}(G)$. We will denote by $\operatorname{ap}(A)$ (resp. wap $(A))$ the set of all almost periodic (resp. weakly almost periodic) functionals on $A$. Both $\operatorname{ap}(A)$ and wap $(A)$ are norm-closed $A$ submodules of $A^{*}$. As is known [2], $\operatorname{ap}\left(L^{1}(G)\right)=\operatorname{AP}(G)$ and $\operatorname{wap}\left(L^{1}(G)\right)=$ $\operatorname{WAP}(G)$, where $\operatorname{AP}(G)$ and $\operatorname{WAP}(G)$ are the spaces of almost periodic and weakly almost periodic functions on $G$ respectively.

We can endow $A^{* *}$ with a product (making $A^{* *}$ a Banach algebra) which is a natural extension of the original product in $A$ (cf. [1]). This product is defined as follows: If $\varphi \in A^{*}$ and $F, H \in A^{* *}$, then we set $\langle F \circ H, \varphi\rangle=$ $\langle F, H \cdot \varphi\rangle$, where $H \cdot \varphi$ is the functional on $A$ defined by $\langle H \cdot \varphi, a\rangle=\langle H, \varphi \cdot a\rangle$, $a \in A$. The algebra $A$ is said to be Arens regular if $A^{* *}$ is commutative. This is equivalent to the condition that $\operatorname{wap}(A)=A^{*}$ (see [1]).

Let $\mu$ be an arbitrary bounded regular Borel measure on $M_{A}$. Then $\mu$ can be considered as an element of $A^{*}$ with respect to the duality

$$
\langle\mu, a\rangle=\int_{M_{A}} \widehat{a}(\phi) d \mu(\phi), \quad a \in A .
$$

It is easy to see that $\sigma_{*}(\mu)$ and $\operatorname{supp} \mu$ in the usual terms are the same.

LEMMA 2.1. If $\mu$ is a bounded regular Borel measure on $M_{A}$, then $\mu \in$ $\operatorname{wap}(A)$.

Proof. We follow basically the proof by Dunkl-Ramirez [2], given there for the Fourier algebra. It is enough to show that if $\mu$ is a positive measure on $M_{A}$ with compact support, then the operator $T_{\mu}$ is weakly compact. Define the map $S: L^{2}\left(M_{A}, d \mu\right) \rightarrow A^{*}$ by $S f=f(\phi) d \mu(\phi)\left(\phi \in M_{A}\right)$. We see that $S$ is a weakly compact operator and $T_{\mu}=S \circ \Gamma$. It follows that the operator $T_{\mu}$ is also weakly compact.

The following lemma was proved in [8, Lemma 6.1] for Arens regular Banach algebras.

LEMMA 2.2. If $A$ has a bounded approximate identity, then every $\varphi \in$ $\operatorname{wap}(A)$ (resp. $\varphi \in \operatorname{ap}(A))$ can be represented as $\varphi=\psi \cdot$ a for some $\psi \in$ $\operatorname{wap}(A)(\operatorname{resp} . \psi \in \operatorname{ap}(A))$ and $a \in A$. 
Proof. Let $\varphi \in \operatorname{wap}(A)$. Note that $\operatorname{wap}(A)$ is a Banach $A$-module. It follows from the Cohen-Hewitt Factorization Theorem [5, 32.22] that the set $\{\psi \cdot a: \psi \in \operatorname{wap}(A), a \in A\}$ is a norm-closed linear subspace of $A^{*}$. Let $\left(e_{i}\right)_{i \in I}$ be a bounded approximate identity for $A$. Then $\varphi \cdot e_{i} \rightarrow \varphi$ in the $w^{*}$-topology. On the other hand, since the set $\left\{\varphi \cdot e_{i}: i \in I\right\}$ is relatively weakly compact, $\varphi \cdot e_{i} \rightarrow \varphi$ weakly. Hence we have

$$
\varphi \in \overline{\{\psi \cdot a: \psi \in \operatorname{wap}(A), a \in A\}}^{w}=\{\psi \cdot a: \psi \in \operatorname{wap}(A), a \in A\} .
$$

For $\operatorname{ap}(A)$ a similar argument works.

A Banach $G$-module $X$ is a Banach space $X$ which is a $G$-module such that:

(i) $e \cdot x=x$ for all $x \in X$, where $e$ is the identity of $G$.

(ii) $\|g \cdot x\| \leq C\|x\|$ for some constant $C>0$, for all $x \in X$ and $g \in G$.

(iii) For all $x \in X$, the map $g \mapsto g \cdot x$ is continuous from $G$ into $X$.

In this case, we can define for each $\varphi \in X^{*}, g \in G$, the element $g \cdot \varphi \in X^{*}$ by $\langle g \cdot \varphi, x\rangle=\langle\varphi, g \cdot x\rangle, x \in X$. A Banach $G$-module $X$ is said to be almost periodic if the set $\{g \cdot x: g \in G\}$ is relatively compact for every $x \in X$. It follows from the Peter-Weyl theory [11, Chap. 4, Sect. 3] that, if $X$ is an almost periodic Banach $G$-module, then $X$ is generated by the eigenvectors of $G$, i.e., by those $x \in X$ that satisfy $g \cdot x=\chi(g) x$ for some $\chi \in \widehat{G}$ and for all $g \in G$.

Lemma 2.3. If there exists a continuous homomorphism $h: L^{1}(G) \rightarrow A$ with dense range, then $A$ is a Banach $G$-module and furthermore $\langle g \cdot \varphi, a\rangle=$ $(\varphi \cdot a)^{\vee}(g)$ for every $\varphi \in A^{*}, a \in A$ and $g \in G$.

Proof. Let $g \in G$ and $f \in L^{1}(G)$. Define $g \cdot h(f)=h\left(f_{g}\right)$, where $f_{g}(s)=$ $f(s-g)$. Let $\left(f_{i}\right)_{i \in I}$ be an approximate identity in $L^{1}(G)$ bounded by one. Since $h\left(\left(f_{i}\right)_{g}\right) h(f) \rightarrow g \cdot h(f)$, we have $\|g \cdot h(f)\| \leq\|h\|\|h(f)\|$. Thus since $h\left(L^{1}(G)\right)$ is dense in $A$ the module operation can be extended to all $A$, after which the algebra $A$ becomes a Banach $G$-module. Now let $\varphi \in A^{*}$ and $a \in A$ be given. It is easy to verify that $\int_{G} f(g)(g \cdot \varphi) d g=\varphi \cdot h(f)$. Using this we have

$$
\int_{G} f(g)\langle g \cdot \varphi, a\rangle d g=\langle\varphi \cdot h(f), a\rangle=\left\langle(\varphi \cdot a)^{\vee}, f\right\rangle .
$$

Since this is true for all $f \in L^{1}(G)$, we obtain

$$
\langle g \cdot \varphi, a\rangle=(\varphi \cdot a)^{\vee}(g) .
$$

Let $A$ be an arbitrary commutative Banach algebra. If $\phi \in M_{A}$ then $\phi \cdot a=\widehat{a}(\phi) \phi$, and consequently, $\phi \in \operatorname{ap}(A)$. Hence, $\overline{\operatorname{span}} M_{A} \subseteq \operatorname{ap}(A)$.

Lemma 2.4. If there exists a continuous homomorphism $h: L^{1}(G) \rightarrow A$ with dense range, then $\operatorname{ap}(A)=\overline{\operatorname{span}} M_{A}$. 
Proof. Let $\varphi \in \operatorname{ap}(A)$. By Lemma 2.2, $\varphi$ is of the form $\varphi=\psi \cdot a$ for some $\psi \in \operatorname{ap}(A)$ and $a \in A$. Since the set $\{g \cdot a: g \in G\}$ is bounded, from the identity $g \cdot \varphi=\psi \cdot(g \cdot a)$ we deduce that the set $\{g \cdot \varphi: g \in G\}$ is relatively compact. On the other hand, since the map $g \mapsto g \cdot \varphi$ is $w^{*}$-continuous, it follows that $g \mapsto g \cdot \varphi$ is norm-continuous. Thus ap $(A)$ is an almost periodic Banach $G$-module. Hence, $\operatorname{ap}(A)$ is generated by the eigenvectors of $G$. Let us now find the eigenvectors of $G$. Assume that $g \cdot \psi=\bar{\chi}(g) \psi$ for some $\chi \in \widehat{G}, \psi \in A^{*} \backslash\{0\}$ and for all $g \in G$. Then for any $f \in L^{1}(G)$, we can write

$$
\psi \cdot h(f)=\int_{G} f(g)(g \cdot \psi) d g=\widehat{f}(\chi) \psi .
$$

It follows that $\chi \in \operatorname{sp}(h)$. Since $h\left(L^{1}(G)\right)$ is dense in $A$, we have $\psi \cdot a=\phi(a) \psi$ for some $\phi \in M_{A}$ and all $a \in A$. Thus since $\operatorname{ker} \phi \subseteq \operatorname{ker} \psi$, we obtain $\psi=c \phi$ for some $c \neq 0$. The proof is complete.

3. The results. The first main result of this note is the following theorem.

THEOREM 3.1. Assume that there exists a continuous homomorphism $h: L^{1}(G) \rightarrow A$ with dense range. If $\varphi \in \overline{\operatorname{span}} \sigma_{*}(\varphi)$ for every $\varphi \in \operatorname{wap}(A)$ with compact spectra, then $M_{A}$ is scattered.

Proof. It suffices to show that every compact subset of $M_{A}$ is scattered. Let $\mu$ be an arbitrary bounded regular Borel measure on $M_{A}$. Then $\mu$ can be considered as a measure on $\widehat{G}$ with $\operatorname{supp} \mu \subseteq \operatorname{sp}(h)$. First we claim that $\mu^{\vee}(g)=\widehat{\mu}(-g)$, where $\widehat{\mu}$ is the Fourier-Stieltjes transform of $\mu$. To see this, let $f \in L^{1}(G)$. Then we can write

$$
\begin{aligned}
\left\langle h^{*} \mu, f\right\rangle & =\langle\mu, h(f)\rangle=\int_{\widehat{G}} \widehat{f}(\chi) d \mu(\chi)=\int_{\widehat{G}}\left(\int_{G} f(g) \bar{\chi}(g) d g\right) d \mu(\chi) \\
& =\int_{G}\left(\int_{\widehat{G}} \bar{\chi}(g) d \mu(\chi)\right) f(g) d g=\int_{G} \widehat{\mu}(g) f(g) d g .
\end{aligned}
$$

Since this is true for all $f \in L^{1}(G)$, we obtain $\mu^{\vee}(g)=\widehat{\mu}(-g)$.

Now let $K$ be an arbitrary compact subset of $M_{A}$ and $\mu$ be an arbitrary continuous regular Borel measure supported on $K$. To prove that $K$ is scattered, in view of $[7$, p. 52 , Theorem 10] it is enough to show that $\mu$ is identically zero. By Lemma $2.1, \mu \in \operatorname{wap}(A)$. Hence, by the assumption we have $\mu \in \overline{\operatorname{span}} K$. It follows that $\mu^{\vee}(g)$ can be approximated in the $\|\cdot\|_{\infty}$ norm by linear combinations of the characters in $K$. Consequently, $\widehat{\mu}(-g)$ is an almost periodic function on $G$. Let $\Phi$ be the invariant mean on $\operatorname{AP}(G)$. Since $\langle\Phi, \chi(g)\rangle=1$ if $\chi=1$ and $\langle\Phi, \chi(g)\rangle=0$ if $\chi=0$, we have

$$
\langle\Phi, \bar{\chi}(g) \widehat{\mu}(-g)\rangle=\mu\{\chi\}=0 .
$$


This shows that all Fourier-Bohr coefficients of the function $\widehat{\mu}(-g)$ are zero. By the uniqueness theorem we obtain $\widehat{\mu}(-g) \equiv 0$, and so $\mu=0$. This proves the theorem.

The next theorem is the second main result of this note.

THEOREM 3.2. Assume that there exists a continuous homomorphism $h: L^{1}(G) \rightarrow A$ with dense range. If the $w^{*}$-spectrum of $\varphi \in \operatorname{wap}(A)$ is compact and scattered, then $\varphi \in \overline{\operatorname{span}} \sigma_{*}(\varphi)$.

Before giving the proof of this theorem, we need the following facts. For $\chi \in \widehat{G}, C_{\chi}[f]$ denotes the Fourier-Bohr coefficient of a function $f \in \operatorname{AP}(G)$. As is known, $C_{\chi}[f]=\int_{\Sigma} \bar{f}(\sigma) d \sigma$, where $\Sigma$ is the Bohr compactification of $G$ and $\bar{f}(\sigma)$ the Bohr extension of $f$. The Bohr spectrum $\sigma_{\mathrm{B}}(f)$ of $f \in \operatorname{AP}(G)$ is defined as the set of all $\chi \in \widehat{G}$ such that $C_{\chi}[f] \neq 0$. We also note that if $f \in \operatorname{AP}(G)$ and $k \in L^{1}(G)$, then $f * k \in \operatorname{AP}(G)$ and $C_{\chi}[f * k]=\widehat{k}(\chi) C_{\chi}[f]$. It is well known that if $f \in \operatorname{AP}(G)$, then $\sigma_{\mathrm{B}}(f) \subseteq \sigma_{*}(f)$ and moreover ${\overline{\sigma_{\mathrm{B}}(f)}}^{*}=\sigma_{*}(f)$.

We also need the following lemma.

Lemma 3.3. If there exists a continuous homomorphism $h: L^{1}(G) \rightarrow A$ with dense range, then $\sigma_{*}\left(\varphi^{\vee}\right) \subseteq \sigma_{*}(\varphi)$ for every $\varphi \in A^{*}$.

Proof. Let $\varphi \in A^{*}$. Suppose that there is $\chi_{0} \in \sigma_{*}\left(\varphi^{\vee}\right)$ but $\chi_{0} \notin \sigma_{*}(\varphi)$. Then there exists a $k \in L^{1}(G)$ such that $\widehat{k}\left(\chi_{0}\right) \neq 0$ and $\widehat{k}=0$ on some neighborhood of $\sigma_{*}(\varphi)$. Let $\pi: A \rightarrow A / I_{\varphi}$ be the canonical map and $\bar{h}=$ $\pi \circ h$. Then $\operatorname{sp}(\bar{h})=\sigma_{*}(\varphi)$. It follows that $k$ belongs to the smallest ideal in $L^{1}(G)$ whose hull is $\operatorname{sp}(\bar{h})$. Hence $\bar{h}(k)=0$, so that $h(k) \in I_{\varphi}$. Consequently, $\varphi \cdot h(k)=0$. It follows from the relation $\varphi^{\vee} * k=h^{*}(\varphi \cdot h(k)$ ) (which can readily be verified) that $k \in I_{\varphi^{\vee}}$. Since $\chi_{0} \in \sigma_{*}\left(\varphi^{\vee}\right)$, we obtain $\widehat{k}\left(\chi_{0}\right)=0$. This is a contradiction.

Proof of Theorem 3.2. Assume that the $w^{*}$-spectrum of $\varphi \in \operatorname{wap}(A)$ is compact and scattered. By Lemma 3.3, $\sigma_{*}\left(\varphi^{\vee}\right)$ is also compact and scattered. The Loomis theorem implies that $\varphi^{\vee} \in \operatorname{AP}(G)$. Since $(\varphi \cdot h(f))^{\vee}$ $=\varphi^{\vee} * f$, we have $\varphi^{\vee} * f \in \operatorname{AP}(G)$ for all $f \in L^{1}(G)$. Also since $h\left(L^{1}(G)\right)$ is dense in $A$, this clearly implies that $(\varphi \cdot a)^{\vee} \in \operatorname{AP}(G)$ for all $a \in A$.

Now let $F \in A^{* *}$ be such that $F(\chi)=0$ for all $\chi \in \sigma_{*}(\varphi)$. To prove the theorem, it suffices to show that $F(\varphi)=0$. Let $\Sigma$ be the Bohr compactification of $G$, and $\bar{f}(\sigma)(\sigma \in \Sigma)$ the Bohr extension of a function $f \in \operatorname{AP}(G)$. For any given $\sigma \in \Sigma$, define $\sigma \cdot \varphi \in A^{*}$ as follows: Since $G$ is dense in $\Sigma$, there exists a net $\left(g_{\lambda}\right)_{\lambda \in \Lambda}$ in $G$ such that $g_{\lambda} \rightarrow \sigma$ in $\Sigma$. Taking into account Lemma 2.3, we can write

$$
\lim _{\lambda}\left\langle g_{\lambda} \cdot \varphi, a\right\rangle=\lim _{\lambda}(\varphi \cdot a)^{\vee}\left(g_{\lambda}\right)=\overline{(\varphi \cdot a)^{\vee}}(\sigma), \quad a \in A .
$$


Since the set $\left\{g_{\lambda} \cdot \varphi\right\}_{\lambda \in \Lambda}$ is bounded, we can define $\sigma \cdot \varphi \in A^{*}$ by

$$
\sigma \cdot \varphi=w^{*}-\lim _{\lambda} g_{\lambda} \cdot \varphi
$$

Note that

$$
\langle\sigma \cdot \varphi, a\rangle=\overline{(\varphi \cdot a)^{\vee}}(\sigma), \quad a \in A
$$

By Lemma 2.2, $\varphi$ can be represented as $\varphi=\psi \cdot a$ for some $\psi \in \operatorname{wap}(A)$ and $a \in A$. Since the set $\{g \cdot a: g \in G\}$ is bounded, from the identity $g \cdot \varphi=$ $\psi \cdot(g \cdot a)$ we deduce that the set $\{g \cdot \varphi: g \in G\}$ is relatively weakly compact. Using (3.1) we get

$$
\sigma \cdot \varphi=w-\lim _{\lambda} g_{\lambda} \cdot \varphi
$$

Hence, the set $\{\sigma \cdot \varphi: \sigma \in \Sigma\}$ is relatively weakly compact (since $\Sigma$ is compact, this set is relatively norm-compact). Using relation (3.2) in the same way, we can see that the map $\sigma \mapsto \sigma \cdot \varphi$ is weakly continuous on $\Sigma$. Therefore, the function $g \mapsto\langle F, g \cdot \varphi\rangle$ is in $\operatorname{AP}(G)$. We claim that $\langle F, g \cdot \varphi\rangle=(F \cdot \varphi)^{\vee}(g)$. To see this, let $f \in L^{1}(G)$. Since the map $g \mapsto g \cdot \varphi$ is weakly continuous, we have

$$
\begin{aligned}
\int_{G}\langle F, g \cdot \varphi\rangle f(g) d g & =\left\langle F, \int_{G}(g \cdot \varphi) f(g) d g\right\rangle=\langle F, \varphi \cdot h(f)\rangle \\
& =\langle F \cdot \varphi, h(f)\rangle=\left\langle(F \cdot \varphi)^{\vee}, f\right\rangle .
\end{aligned}
$$

Since this is true for all $f \in L^{1}(G)$, our claim follows. We also note that

$$
\langle F, \sigma \cdot \varphi\rangle=\overline{(F \cdot \varphi)^{\vee}}(\sigma), \quad \sigma \in \Sigma .
$$

Let us now find the Fourier-Bohr coefficients of the function $(F \cdot \varphi)^{\vee}$. For this purpose consider the following vector-valued integral:

$$
\varphi_{\chi}=\int_{\Sigma} \bar{\chi}(\sigma)(\sigma \cdot \varphi) d \sigma .
$$

Then we have

$$
\begin{aligned}
\left\langle\varphi_{\chi}, a\right\rangle & =\int_{\Sigma} \bar{\chi}(\sigma)\langle\sigma \cdot \varphi, a\rangle d \sigma=\int_{\Sigma} \bar{\chi}(\sigma) \overline{(\varphi \cdot a)^{\vee}}(\sigma) \\
& =C_{\chi}\left[(\varphi \cdot a)^{\vee}\right]=\widehat{a}(\chi) C_{\chi}\left[\varphi^{\vee}\right], \quad a \in A .
\end{aligned}
$$

It follows that $\varphi_{\chi}=\chi C_{\chi}\left[\varphi^{\vee}\right]$. Further, since the mapping $\sigma \mapsto \sigma \cdot \varphi$ is weakly continuous, in view of (3.3) we obtain

$$
\begin{aligned}
C_{\chi}\left[(F \cdot \varphi)^{\vee}\right] & =\int_{\Sigma} \bar{\chi}(\sigma) \overline{(F \cdot \varphi)^{\vee}}(\sigma) d \sigma=\int_{\Sigma} \bar{\chi}(\sigma)\langle F, \sigma \cdot \varphi\rangle d \sigma \\
& =\left\langle F, \varphi_{\chi}\right\rangle=F(\chi) C_{\chi}\left[\varphi^{\vee}\right] .
\end{aligned}
$$

Since $\sigma_{*}\left(\varphi^{\vee}\right) \subseteq \sigma_{*}(\varphi)$ and $F(\chi)=0$ for all $\chi \in \sigma_{*}(\varphi)$, it follows that all Fourier-Bohr coefficients of the function $(F \cdot \varphi)^{\vee}$ are zero. By the uniqueness 
theorem, $(F \cdot \varphi)^{\vee} \equiv 0$. In particular, we have $F(\varphi)=(F \cdot \varphi)^{\vee}(e)=0$. This proves the theorem.

Let us record a consequence of this theorem.

COROLlary 3.4. Assume that there exists a continuous homomorphism $h: L^{1}(G) \rightarrow A$ with dense range and $M_{A}$ is scattered. Then the algebra $A$ is Arens regular if and only if $A^{*}=\overline{\operatorname{span}} M_{A}$.

Proof. It is clear that if $A^{*}=\overline{\operatorname{span}} M_{A}$, then $A$ is Arens regular. Now assume that $A$ is Arens regular. Then $\operatorname{wap}(A)=A^{*}$ by Theorem 1 of [1]. Let $\varphi \in A^{*}$. By Lemma 6.1 of [8] (see also Lemma 2.2 of this note), $\varphi$ is of the form $\varphi=\psi \cdot a$ for some $\psi \in A^{*}$ and $a \in A$. It suffices to show that $\psi \cdot h(f) \in \overline{\operatorname{span}} M_{A}$ for every $f \in L^{1}(G)$. Let $\left(f_{i}\right)_{i \in I}$ be a bounded approximate identity for $L^{1}(G)$ such that supp $\widehat{f}_{i}(i \in I)$ is compact. Then $\left(h\left(f_{i}\right)\right)_{i \in I}$ is a bounded approximate identity for $A$. Since $\sigma_{*}(\psi)$ is scattered, from the relation

$$
\sigma_{*}\left(\psi \cdot h(f) h\left(f_{i}\right)\right) \subseteq \sigma_{*}(\psi) \cap \operatorname{supp} \widehat{f} \cap \operatorname{supp} \widehat{f_{i}}
$$

(which can be readily verified), we deduce that $\sigma_{*}\left(\psi \cdot h(f) h\left(f_{i}\right)\right)$ is compact and scattered. By Theorem 3.2, we have $\psi \cdot h(f) h\left(f_{i}\right) \in \overline{\operatorname{span}} M_{A}$. Since $\psi \cdot h(f) h\left(f_{i}\right) \rightarrow \psi \cdot h(f)$ in norm, we obtain $\psi \cdot h(f) \in \overline{\operatorname{span}} M_{A}$. The proof is complete.

\section{References}

[1] J. Duncan and S. A. R. Hosseiniun, The second dual of a Banach algebra, Proc. Roy. Soc. Edinburgh Sect. A 84 (1979), 309-325.

[2] C. F. Dunkl and D. E. Ramirez, Weakly almost periodic functionals on the Fourier algebra, Trans. Amer. Math. Soc. 185 (1973), 501-514.

[3] P. Głowacki, On functions with scattered spectra on lca groups, Studia Math. 70 (1981), 147-152.

[4] C. Herz, Harmonic synthesis for subgroups, Ann. Inst. Fourier (Grenoble) 23 (1973), no. 3, 91-123.

[5] E. Hewitt and K. Ross, Abstract Harmonic Analysis II, Springer, 1970.

[6] J. W. Kitchen, Jr., Normed modules and almost periodicity, Monatsh. Math. 70 (1966), 233-243.

[7] H. E. Lacey, The Isometric Theory of Classical Banach Spaces, Springer, 1974.

[8] A. T.-M. Lau and A. Ülger, Some geometric properties on the Fourier and FourierStieltjes algebras of locally compact groups, Arens regularity and related problems, Trans. Amer. Math. Soc. 337 (1993), 321-359.

[9] L. H. Loomis, The spectral characterization of a class of almost periodic functions, Ann. of Math. 72 (1960), 362-368.

[10] F. Lust-Piquard, Means on $C V_{p}(G)$-subspaces of $C V_{p}(G)$ with $R N P$ and Schur property, Ann. Inst. Fourier (Grenoble) 39 (1989), 969-1006.

[11] Y. I. Lyubich, Introduction to the Theory of Banach Representation Groups, Birkhäuser, 1988. 
[12] G. S. Mustafaev, Banach algebras with bounded groups of generators, and the Schur property, Math. Notes 71 (2002), 661-666.

[13] W. Żelazko, Banach Algebras, PWN and Elsevier, Warszawa and Amsteram, 1973.

H. S. Mustafayev

Institute of Mathematics and Mechanics

National Academy of Sciences of Azerbaijan

F. Agaev St. 9

Baku, Azerbaijan

E-mail: hsmustafayev@yahoo.com
Current address: Department of Mathematics Faculty of Arts and Sciences Yuzuncu Yil University 65080 Van, Turkey

Received June 30, 2004 